\title{
Estado innovador de empresas medianas y pequeñas agroindustriales de Manizales y su relación con el desempeño organizacional ${ }^{*}$
}

\author{
Innovative state of small and medium sized agro-industries \\ of Manizales and their relation with the organizational performance \\ Estado inovador de empresas agroindustriais medianas e pequenas \\ de Manizales e sua relação com o desempenho organizacional
}

Recibido el 17 de noviembre de 2016. Aceptado el 6 de febrero de 2017

\author{
Natalia Andrea Toro Zuluaga** \\ Colombia \\ Juan Manuel Castaño Molano*** \\ Colombia \\ Yurani López Espitia**** \\ Colombia
}

Para citar este artículo:

Toro Zuluaga, Natalia Andrea,

Castaño Molano, Juan Manuel

y López Espitia, Yurani (junio,

2017). Estado innovador de empresas medianas y pequeñas agroindustriales de Manizales y su relación con el desempeño organizacional. Ánfora, 24(42),

67 - 93. Universidad Autónoma de Manizales. ISSN 0121-6538.

\section{Resumen}

Objetivos: identificar el estado de innovación de las pymes agroindustriales de Manizales y su relación con el desempeño organizacional. Metodología: a partir de la identificación de la innovación que prevalece en las empresas objeto deestudio, la percepción de desempeño expresada por los empresarios encuestados y la relación

\footnotetext{
*Artículo resultado de la investigación Impacto de la Innovación en el desempeño de pymes agroindustriales de la ciudad de Manizales. Universidad Católica Luis Amigó. Presentado y aprobado en el mes de febrero 2015 y culminado en el mes de octubre de 2016.

** Especialista en Gerencia de Negocios Internacionales. Universidad Nacional de Colombia, sede Manizales. Correo electrónico: ntoroz@unal.edu.co

*** Magister en Administración Economista Empresarial. Universidad Nacional, sede Manizales. Correo electrónico: jmcastanom@unal.edu.co

**** Magister en Gerencia del Talento Humano. Profesora Universidad Católica Luis Amigo. Correo electrónico: mz_administracion@funlam.edu.co
} 
entre ambas variables, el diseño metodológico se basó en cinco etapas: formulación del problema, construcción del modelo teórico-metodológico, trabajo de campo y análisis de la información cuantitativa. La contrastación se llevó a cabo en 12 empresas con la aplicación de dos cuestionarios: estado de innovación y percepción de desempeño.

Resultados: las 12 empresas presentaron una innovación enfocada en procesos relacionados con la actualización o renovación de equipos, seguido de la innovación de productos y, luego, en innovación organizacional con cambios significativos en la gestión. Se halló que existe una relación positiva entre el estado innovador identificado y el desempeño organizacional. Conclusiones: la mayoría de las empresas introducen factor innovador para su crecimiento y posicionamiento en el mercado, procurando la innovación en procesos y asuntos organizacionales.

Palabras clave: Innovación; Agroindustria; Desempeño organizacional.

\section{Abstract}

Objectives: to identify the innovative state of SMEs agro-industries of Manizales and their relationship with the organizational performance. Methodology: taking into account the innovation prevailing within the companies under study, the performance perception and the relationship between the two variables, the methodological design was based onfivestages asfollows: problemformulation, theoretical and methodological construction of the model; fieldwork and quantitative information analysis. The comparison was conducted in 12 agro-industries by applying two questionnaires: innovative state and performance perception. Results: the 12 participating agroindustries exhibited an innovation focused on processes, particularly in relation to equipment upgrading or renewing, followed by -product innovation -product Improvements or adjustments. In relation to organizational innovation, they showed significant changes in management. Findings showed a positive relationship between the innovative state and organizational performance. Conclusions: most companies implement an innovative factor for their growth and market positioning, ensuring innovation in processes for their continuity within the competitive environment.

Keywords: Innovation; Agro-industries; Organizational Performance. 


\section{Resumo}

Objetivos: identificar o estado de inovação nas pymes agroindustriais de Manizales e sua relação com o desempenho organizacional. Metodologia: a partir da identificação da inovação prevalecente nas empresas sob estudo, a percepção do desempenho expressada pelos empresários pesquisados e a relação entre as duas variáveis, o desenho metodológico foi baseado em cinco fases: formulação do problema, a construção do modelo teórico-metodológico, trabalho de campo e análise da informação quantitativa. O contraste foi realizado em 12 empresas com a aplicação de dois questionários: estado da inovação e percepção de desempenho. Resultados: as 12 empresas apresentaram uma inovação focada em processos relacionados com a atualização ou renovação de equipamentos, seguido da inovação de produtos e, em seguida, inovação organizacional com mudanças significativas nos processos de gestão. Achou-se que existe uma relação positiva entre o estado inovador identificado e o desempenho organizacional. Conclusões: a maioria das empresas introduzem o fator inovador para seu crescimento e posicionamento no mercado, garantindo a inovação em processos e questões organizacionais.

Palavras-chave: Inovação; agroindústria; desempenho organizacional. 


\section{Introducción}

Desde el inicio de la apertura económica colombiana de los años 90, las empresas se han propuesto ser más competitivas, aplicando procesos de modernización tecnológica, organización de la producción, en la gestión de los recursos humanos (Cárdenas, 1999) o por bajos precios; esta última es una estrategia limitada y fácil de imitar; lo cual ha hecho que las empresas reconozcan que aquellas centradas en racionalización de costos, se agotaron y requieren estrategias innovadoras (Cardona, Castiblanco y Díaz, 2013).

En este panorama, los avances de la globalización y el actual contexto tecnológico requieren que la innovación se convierta en factor competitivo para las empresas, a través del aprovechamiento de sus recursos y capacidades (Coronado, Echeverri y Arias, 2014). Para Cortés (2007) la agroindustria es un factor clave para promover la capacidad empresarial, a partir de la innovación y el desarrollo tecnológico; esto, con el fin de mejorar las condiciones productivas al integrar el campo con la ciudad, generando alianzas estratégicas entre el sector privado y público, que permitan lograr rendimientos y eficiencia industrial con productos de exportación.

Ovalle, Martínez, Restrepo y Ramírez (2012) en su estudio sobre el sector textil plantean los tres niveles de innovación en las cuales se encuentran las pymes; muestran que el $67 \%$ tiene un nivel de innovación en la fase de elaboración de proyectos; el 13\% se encuentra en un nivel intermedio es decir, en la etapa estratégica de innovación, y el 20\% restante está en nivel de cultura innovadora; Zartha, Avalos, Aguilar y Castrillón (2009) afirman, frente al sector agroindustrial, que la innovación debe tener mayor desarrollo, dado que de 1227 empresas estudiadas el 9\% fueron clasificadas como innovadoras radicales; el 20\% como innovadoras incrementales, el $7 \%$ como innovadoras organizacionales; el $43 \%$ como adecuadas tecnológicamente y el $21 \%$ como no innovadoras.

Igualmente, Medina, Vélez, Zartha, Ruiz y Copete (2012) indican que de 21 empresas de este sector para departamento de Antioquia, el 50\% son consideradas potencialmente innovadoras en producto-servicio, no obstante, la innovación en procesos es la dimensión que más carencias y menos desarrollo presenta.

Ahora bien, el concepto de agroindustria, según la Organización de las Naciones Unidas para Alimentación y la Agricultura FAO (1997) se define como aquel sector manufacturero cuya materia prima y productos están relacionados con la transformación de productos agrícolas, pesqueros o forestales; es decir, la 
fabricación de alimentos, bebidas y tabaco, textiles, muebles y demás productos de madera, papel y su procesamiento y caucho, entre otros.

Para Ickis, Leguizamon Metzgerg y Flores (2009) la agroindustria es una actividad que se centra en el procesamiento de insumos agropecuarios para la elaboración de productos que satisfagan expectativas de consumidores y responde a tres momentos industriales: adquisición -extracción de materia prima del campo-, transformación y comercialización -distribución del producto en el mercado objetivo- (Austin, 1992; Cortés, Suárez y Pardo, 2008; Planella, 1981; Argoti, 2009). De acuerdo con la Clasificación Industrial Internacional Uniforme (CIIU) la agroindustria se clasifica en: Alimentos, Bebidas y Tabaco, Textil: prendas de vestir y cueros, Madera, Papel y actividades de edición e impresión y Caucho.

En Colombia, el renglón económico agrícola ha contribuido al desarrollo nacional, al ser considerado un factor de oportunidad para invertir, en el que los sectores agroindustriales como carne bovina, chocolatería confitería, palma, aceites y grasas se ubican en el nivel de talla mundial (Proexport, 2012) ${ }^{1}$. El Plan Nacional de Desarrollo Colombiano del periodo 2014-2018 $8^{2}$ enuncia el protagonismo del sector agropecuario, reflejado en esfuerzos para la promoción de políticas públicas, apoyo a la planificación rural y asignación y ejecución de mayores presupuestos en el sector; igualmente, con actividades encaminadas al mejoramiento de la infraestructura y uso eficiente y equitativo de los suelos para el logro de sistemas productivos rentables y competitivos (Departamento Nacional de Planeación [DPN], 2015) a través de la investigación y desarrollo tecnológico (Flórez, 2013).

Para el Departamento Nacional de Estadística DANE (2016) este sector aporta aproximadamente el 6,1\% del PIB y genera un promedio del $16,3 \%$ del empleo nacional, convirtiéndose en una fuente de recursos naturales y de ventajas comparativas del país. Esto se refleja en los renglones de cultivo de café, de otros productos agrícolas, producción pecuaria y silvicultura; en este ámbito los departamentos con mayores índices de participación: Boyacá (20,1\%); Cundinamarca (15\%); Nariño (13,1\%) y Cauca (11,6\%). Es de resaltar que de los tres departamentos que conforman el paisaje cafetero en Colombia-Caldas, Risaralda y Quindío- Caldas obtiene el mejor desempeño con un promedio de $1.7 \%$ de participación en unidades productoras.

1 Actualmente Procolombia.

2 "Todos por un nuevo país" 
Específicamente, en el Departamento de Caldas se estableció un proyecto de distritos agroindustriales que se dividen en seis subregiones (ver figura 1), los cuales son considerados como una estrategia de integración por regiones para dar respuesta al desarrollo económico, a partir de las ventajas propias y de la articulación de cadenas productivas (Plan de Desarrollo Departamental, 2001$2003)^{3}$.

Figura 1. Distritos Agroindustriales del Departamento de Caldas

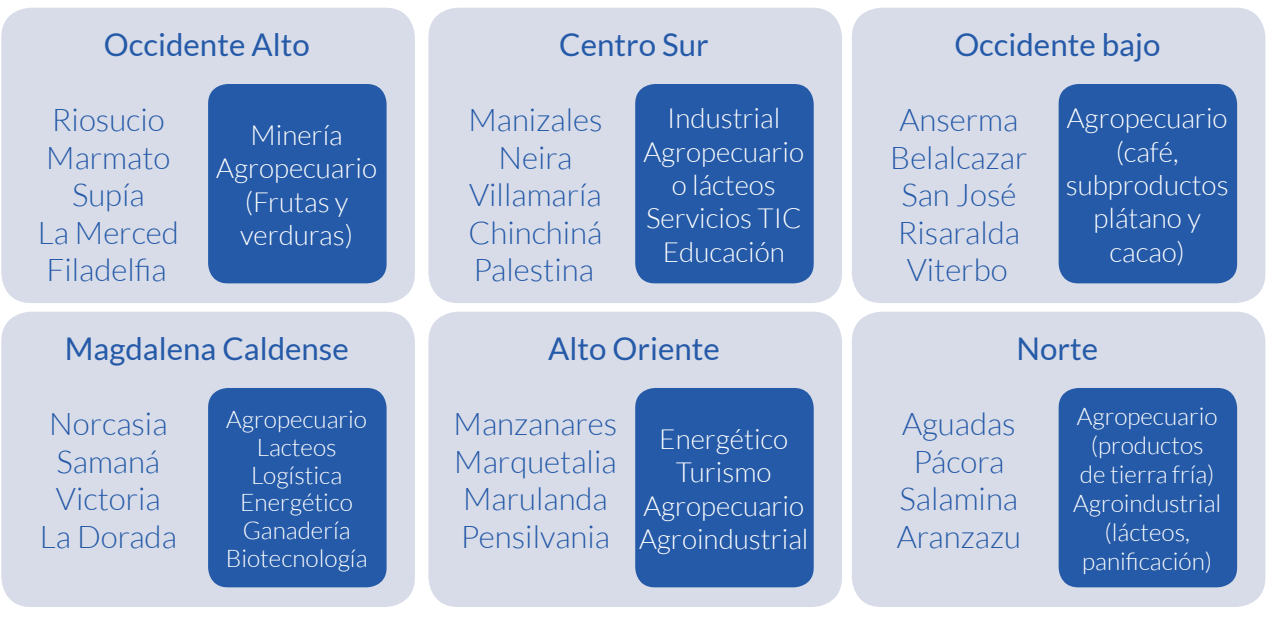

Fuente: Gobernación de Caldas 2001

Así, los distritos agroindustriales permiten vislumbrar las potencialidades en la producción agrícola caracterizada, principalmente, por cultivos de café, plátano y cacao, seguido del desarrollo ganadero en algunos sectores y en medidas menores el turismo, los servicios y la industria. De allí que la Agenda Interna para la Productividad y la Competitividad de Caldas indique que el Departamento debe asumir la inversión en productividad e infraestructura para la agroindustria y el mejoramiento de escenarios para atracción de inversión externa, fomento del sector productivo, capitales para financiación, centros de desarrollo tecnológico y productivo que aproveche las condiciones de biodiversidad de la región, mano de obra calificada, experiencia industrial y buena calidad de vida (DPN, 2007). 
Con respecto a la innovación, ésta es considerada como un ajuste resultante de ideas y acciones creativas que impactan la productividad organizacional (Knigth, 1967; Dosi, 1988; Sánchez, García y Mendoza, 2014) a través del aprovechamiento de sus recursos y capacidades (Coronado, Echeverri y Arias, 2014) para fortalecer el rendimiento de las mismas, desarrollando y mejorando sus potencialidades para la creación de nuevos productos, mejora los procesos de producción y la puesta en marcha de prácticas organizativas que permitan la generación de conocimiento (Organización para la Cooperación y el Desarrollo Económico [OCDE], 2005).

La innovación se entiende también como el resultado del aprendizaje y éste es usado para el mejoramiento de los productos o procesos (Dankbaar y Cobbenhagen, 2000). Es decir, la innovación se da a través del uso de nuevo conocimiento tecnológico - componentes, vínculos, procesos y técnicas- o de mercado - conocimiento de canales, preferencias y necesidades del consumidorpara la oferta de un nuevo producto (Afuah, 1998) como motor del crecimiento económico (Rose, Shipp, Lal y Stone, 2009) y generador de valor y bienestar para las sociedades (Varela, Contesse y Silva, 2009).

Para Schumpeter (1934) la innovación es la transformación en las composiciones de los factores de producción, cambios en los métodos, modificaciones en la organización industrial, en la producción de un artículo nuevo o en la apertura de nuevos mercados.

Montoya (2004) y Kóhler y González (2014), basados en la teoría del Desenvolvimiento Económico -Schumpeter en 1912- consideran que la innovación es un factor determinante como proceso que surge de las organizaciones; los responsables son los emprendedores que propenden por los cambios y nuevas combinaciones y elementos, generando la "destrucción creativa" que conlleva a la optimización, dificultad de imitación y desplazamiento de la competencia (Yoguel, Barletta y Pereira, 2013; Cano, Sánchez, González y Pérez, 2014), logrando eficiencia, mejores precios y calidad, constituyendo así un sistema económico dinámico y creciente (Sánchez, 2005).

De allí que la literatura en innovación, referencie dos perspectivas: radical $e$ incremental. La primera, se entiende como aquel resultado que propicia transformaciones determinantes y novedosas -introducción de bienes en el mercado, métodos de producción, apertura de mercados, materias primas y cambios de procesos de gestión- (Montoya, 2004; Parra, Mesa, Corrales y Aguirre, 2007); y la segunda, a mejoras sustanciales, pero de menor impacto 
con ajustes o medidas menores (Knight, 1967; Tushman y Anderson, 1986; Damanpour, 1991; Parra et al. 2007).

No obstante, para el presente trabajo se tomará la clasificación del Manual de Oslo: producto, proceso, mercadeo y organización (OCDE, 2005) semejantes a las que propuso Schumpeter en 1934 en relación introducción de nuevos bienes, introducción de un nuevo método productivo ya existente, apertura de un nuevo mercado, nuevas fuentes de abastecimiento de materias primas y nuevas estructuras de mercado.

En la Tabla 1 se resumen las descripciones de los diferentes estudios mencionados frente a los tipos de innovación.

\begin{tabular}{|c|c|l|}
\hline $\begin{array}{c}\text { Tipo de } \\
\text { innovación }\end{array}$ & Autor & \multicolumn{1}{c|}{ Descripción } \\
\hline \multirow{1}{*}{ Innovación de } \\
producto & $\begin{array}{c}\text { Goldenberg, } \\
\text { yorowitz, Levav } \\
\text { yazursky } \\
(2003)\end{array}$ & $\begin{array}{l}\text { La innovación en productos se da por las mejoras o } \\
\text { ajustes en los atributos y componentes, alcanzando } \\
\text { la satisfacción y logro de objetivos. }\end{array}$ \\
\cline { 2 - 4 } & $\begin{array}{c}\text { Manual de Oslo, } \\
\text { OCDE (2005) }\end{array}$ & $\begin{array}{l}\text { En esta clasificación se encuentra la introducción o } \\
\text { cambios significativos en las características de los } \\
\text { bienes y servicios, sean como ejemplo en condicio- } \\
\text { nes funcionales o de uso de los primeros o eficiencia } \\
\text { o rapidez en los segundos. }\end{array}$ \\
\cline { 2 - 4 } & $\begin{array}{l}\text { Zhan y Li } \\
\text { (2012) }\end{array}$ & $\begin{array}{l}\text { Los objetivos para el desarrollo de nuevos productos } \\
\text { está relacionado con el alcance de mayores ingresos, } \\
\text { obtener mayores niveles de visibilidad externa y la } \\
\text { confianza en la operación de nuevos mercados, lo } \\
\text { cual es alineado a la necesidad de las empresas ser } \\
\text { competitivas a través de la innovación. }\end{array}$ \\
\cline { 2 - 3 } & $\begin{array}{l}\text { Teece (1996) } \\
\text { Cano, Sánchez, } \\
\text { González y } \\
\text { Pérez (2014). }\end{array}$ & $\begin{array}{l}\text { Para el desarrollo de nuevos productos es necesa- } \\
\text { rio tener suficiencia en recursos, conocimientos y } \\
\text { capacidades, por ello la selección de los modelos de } \\
\text { innovación deberá estar acorde con los requerimien- } \\
\text { tos y objetivos de las organizaciones, reconociendo } \\
\text { al capital intelectual como elemento trascendental } \\
\text { para la generación de innovación. }\end{array}$ \\
\hline
\end{tabular}




\begin{tabular}{|c|c|c|}
\hline $\begin{array}{c}\text { Tipo de } \\
\text { innovación }\end{array}$ & Autor & Descripción \\
\hline \multirow{5}{*}{$\begin{array}{c}\text { Innovación de } \\
\text { procesos }\end{array}$} & $\begin{array}{l}\text { Davenport } \\
\text { (1993) }\end{array}$ & $\begin{array}{l}\text { La innovación de procesos envuelve nuevas activida- } \\
\text { des laborales, la labor de proceso y la ejecución del } \\
\text { cambio de las condiciones humanas, tecnológicas y } \\
\text { organizacionales. }\end{array}$ \\
\hline & Freire (2000) & $\begin{array}{l}\text { Las innovaciones de proceso se dan en el interior de } \\
\text { la organización, por el contrario las de producto se } \\
\text { orientan hacia el mercado. }\end{array}$ \\
\hline & $\begin{array}{l}\text { Manual de Oslo, } \\
\text { OCDE (2005) }\end{array}$ & $\begin{array}{l}\text { La generación de un nuevo proceso productivo o de } \\
\text { distribución o una mejora significativa en el mismo, } \\
\text { incluye técnicas, materiales, suministros, programas } \\
\text { y equipos, con el fin disminuir costos o mejorar la } \\
\text { calidad. }\end{array}$ \\
\hline & $\begin{array}{l}\text { Coronado, } \\
\text { Echeverri y } \\
\text { Arias (2014) }\end{array}$ & $\begin{array}{l}\text { Dentro de los resultados del estudio de se presentan } \\
\text { indicadores importantes de medir en las innovacio- } \\
\text { nes de proceso tales como: capacidad productiva, } \\
\text { flexibilidad de la producción, eficiencia en costos, } \\
\text { optimización de materias primas, desarrollo de } \\
\text { habilidades de los empleados y patentes de un nuevo } \\
\text { proceso. }\end{array}$ \\
\hline & $\begin{array}{l}\text { Lombardi y } \\
\text { Mora (2014) }\end{array}$ & $\begin{array}{l}\text { La voluntad de las organizaciones por mejorar sus } \\
\text { productos y procesos representan productividad, } \\
\text { adicional a la eficiencia económica que logran las } \\
\text { empresas que invierten en innovación. }\end{array}$ \\
\hline \multirow[t]{2}{*}{$\begin{array}{c}\text { Innovación de } \\
\text { mercadeo }\end{array}$} & $\begin{array}{l}\text { Manual de Oslo, } \\
\text { OCDE (2005) }\end{array}$ & $\begin{array}{l}\text { Nuevos métodos o estrategias de comercialización, } \\
\text { que incluyan ajustes en el diseño (forma y aspecto), } \\
\text { posicionamiento o promoción de productos y que } \\
\text { tratan de satisfacer las necesidades y deseos de los } \\
\text { consumidores. Adicional implica también para este } \\
\text { tipo de innovaciones los nuevos canales de venta y } \\
\text { precios. }\end{array}$ \\
\hline & $\begin{array}{l}\text { Barbosa y Do- } \\
\text { minique (2012) }\end{array}$ & $\begin{array}{l}\text { La innovación en mercadeo se encuentra asociada a } \\
\text { la capacidad de las empresas de descifrar las necesi- } \\
\text { dades, deseos y expectativas del mercado }\end{array}$ \\
\hline
\end{tabular}




\begin{tabular}{|c|c|c|}
\hline $\begin{array}{c}\text { Tipo de } \\
\text { innovación }\end{array}$ & Autor & Descripción \\
\hline \multirow{5}{*}{$\begin{array}{l}\text { Innovación de } \\
\text { organización }\end{array}$} & Evan (1966) & $\begin{array}{l}\text { El autor diferencia la innovación técnica de la admi- } \\
\text { nistrativa, ilustrando que la primera corresponde a } \\
\text { una idea de nuevo producto o proceso, en un sentido } \\
\text { más tecnológico, mientras la segunda consiste en in- } \\
\text { novaciones de la estructura social de la organización, } \\
\text { tales como la formación de las tareas, asignación de } \\
\text { recursos, políticas, entre otros. }\end{array}$ \\
\hline & Daft (1978) & $\begin{array}{l}\text { Las innovaciones a nivel administrativo y técnico, } \\
\text { involucran múltiples acciones a nivel de toma de } \\
\text { decisiones y cambios en las actividades de la organi- } \\
\text { zación, definiendo la innovación organizacional como } \\
\text { la adopción de nuevas ideas o comportamientos por } \\
\text { parte de la organización. }\end{array}$ \\
\hline & $\begin{array}{l}\text { Damanpour y } \\
\text { Evan (1984) }\end{array}$ & $\begin{array}{l}\text { La innovación administrativa incluye la estructura } \\
\text { organizacional y los procesos administrativos dentro } \\
\text { de los esquemas de gestión, más que de las mismas } \\
\text { las tareas básicas }\end{array}$ \\
\hline & $\begin{array}{c}\text { Damanpour } \\
\text { (1991) }\end{array}$ & $\begin{array}{l}\text { La innovación organizativa presenta influencia de los } \\
\text { individuos, de la misma organización y del medio am- } \\
\text { biente, todo pensado para contribuir al rendimiento, } \\
\text { desempeño y a la eficacia de la empresa. }\end{array}$ \\
\hline & $\begin{array}{l}\text { Manual de Oslo, } \\
\text { OCDE (2005) }\end{array}$ & $\begin{array}{l}\text { Este tipo de innovación se presenta cuando se } \\
\text { establecen nuevas prácticas, formas y métodos de } \\
\text { organización en las empresas. }\end{array}$ \\
\hline
\end{tabular}

Ahora, en Colombia la innovación ha sido un factor clave para el desarrollo empresarial, teniendo en cuenta la cantidad de Tratados Libres de Comercio, TLC, que hasta la fecha el país ha firmado con otras naciones (González, 2013); esto exige a las pequeñas y medianas empresas, estar a la vanguardia competitiva. Ortega (2000) afirma que la innovación tecnológica es una salida eficaz para contrastar los problemas económicos que enfrentan las pymes, las cuales a través de procesos y/o productos innovadores dan frente a las exigencias competitivas del entorno, al considerar que aquellas empresas que se dedican a innovar son las que se mantendrán.

Sumado a lo expuesto, es preciso determinar la existencia de modelos para evaluar o determinar el nivel de desempeño. Rotundo y Sackett (2002) sustentan el modelo de comportamientos hacia el objetivo organizacional, entorno social y psicológico y el de comportamientos contraproducentes; es decir, aquellos 
que afectan el bienestar organizacional. Campbell (1990) plantea un modelo de ocho componentes -Suficiencia en tareas específicas del cargo, comportamientos comunes a los cargos (tareas no específicas), comunicación oral y escrita, esfuerzo y voluntad, disciplina, soporte a los colegas, suficiencia en supervisión y capacidad de administración y gerencia.

Para Daft (2007), la efectividad empresarial es definida por el alcance de metas -indicadores de producción, rentabilidad, crecimiento, satisfacción de consumidores, calidad- y de procesos internos -valoración de cultura organizacional, clima laboral, trabajo en equipo, confianza, comunicación, recompensas-. Molnar y Rogers (1976) contemplan que la efectividad responde a la relación existente entre rendimiento y objetivos, el proceso de toma de decisiones como un factor determinante en el desempeño organizacional, la autonomía y la claridad en el objetivo corporativo.

Por su parte, Quinn y Rohbaugh (1983) afirman que la efectividad de las organizaciones depende de los modelos de análisis que se desarrollen en éstas y debe abarcar aspectos como el alcance de los objetivos, los medios y los fines de la organización, la valoración de los recursos humanos y el énfasis en el entrenamiento y el desarrollo que permite medir la efectividad: flexibilidad y adaptación, crecimiento, evaluaciones de entes externos, uso del ambiente, prontitud, ganancias, productividad, planeación de metas, eficiencia, control, estabilidad, gerencia de la información y la comunicación, conflicto vs. cohesión, calidad, moral, valoración de recursos humanos y énfasis en el entrenamiento y el desarrollo.

Gupta y Govindarajan (1984) sostienen que la efectividad debe responder a 13 aspectos significativos en la organización: ventas, tasa de crecimiento, proporción de mercado, rentabilidad operativa, rentabilidad en ventas, flujo de caja de las operaciones, retorno sobre la inversión, desarrollo de nuevos productos, desarrollo de mercados, actividades de I+D, programas de reducción de costos, desarrollo de personal y asuntos políticos y públicos. Para el presente trabajo, se tomará el modelo planteado por Gupta y Govindarajan (1984), debido a su precisión y contrastación empírica.

Respecto a la relación de las variables -innovación y desempeño- García, Gálvez y Maldonado (2016) determinan que la acción innovadora en productos, procesos y sistemas de gestión que promueven las pequeñas y medianas empresas tienen un impacto positivo sobre su crecimiento, es decir, la innovación mejora las capacidades, la competitividad, el desempeño y el rendimiento empresarial de las pymes (Lecerf, 2012 y Wang, 2014). 
Yamakawa y Ostos (2011) concluyen en su estudio que la innovación organizacional impacta al desempeño organizacional al considerar la de tipo técnica -ajustes en productos, servicios, procesos y métodos de produccióncomo medio para alcanzar los objetivos empresariales, más allá de los cambios en estructura y procesos administrativos. Damanpour y Evan (1984) explican que debe haber cambios de estructura y estrategia en las organizaciones a partir de los constantes cambios del entorno, obteniendo un alto desempeño organizacional al balancear la innovación técnica y administrativa.

En este sentido, García, Quintero y Arias (2014) analizan la relación entre las capacidades de innovación, el desempeño innovador y el desempeño organizacional -financiero y no financiero- en empresas de servicios. Su resultado es que las capacidades de innovación relacionadas con el cliente y mercadeo influyen en el desempeño innovador y el desempeño organizacional, lo que significa la importancia de la innovación para el éxito corporativo en mercados dinámicos y cambiantes (Damanpour y Gopalakrishnan, 2001). Por esta razón, las organizaciones deben asumir las innovaciones como adaptaciones a las amenazas y oportunidades del entorno para mejorar y sostener su desempeño (Afcha, 2011) y lograr, a partir de condiciones organizacionales internas, ventajas competitivas y desempeño superior (Camisón y Villar, 2014).

A partir de esta realidad empresarial, el presente trabajo pretende identificar el estado innovador de las pymes agroindustriales de la ciudad de Manizales y su relación con el desempeño organizacional (Goldenberg, Horowitz, Levav y Mazursky, 2003; Zhan y Li, 2012; Teece, 1996; Cano, Sánchez, González y Pérez, 2014; Davenport, 1993; Freire, 2000; Coronado, Echeverri y Arias, 2014; Lombardi y Mora, 2014). La presente investigación se plantea novedosa, útil y de interés en primer lugar por ser un tema de investigación poco abordado; en segundo lugar, será de utilidad para la academia y para el medio socioempresarial fortaleciendo los procesos investigativos de la universidad como al sector empresarial, el cual se verá beneficiado con los hallazgos de la presente investigación para mejorar su gestión y fortalecer su competitividad. Finalmente, es de gran interés dado que permitirá la integración desde la academia con las pequeñas y medianas empresas (pymes) de la ciudad de Manizales. 


\section{Metodología}

Este estudio es de perspectiva cuantitativa. El diseño metodológico comprendió cinco etapas: formulación del problema, construcción del modelo teórico-metodológico (la contrastación se realizó en empresas medianas y pequeñas del sector agroindustrial de Manizales), trabajo de campo, y el análisis de la información cuantitativa (procesamiento y el análisis de la información cuantitativa, para la cual se utilizó regresión canónica para determinar la relación entre innovación y desempeño).

Teniendo en cuenta la definición de Agroindustria propuesta por Argoti (2009), se solicitó a la Cámara de Comercio de Manizales la base de datos de las empresas registradas al año 2016 que incluyeran los códigos de las actividades: Alimentos, Bebidas y Tabaco, Textil, Madera, Papel y Caucho, de acuerdo con la Clasificación Industrial Internacional Uniforme (CIIU).

Tabla 2. Actividades Agroindustriales CIUU

\begin{tabular}{|c|c|}
\hline Código CIUU & Descripción de la actividad \\
\hline \multicolumn{2}{|c|}{ Elaboración de productos alimenticios } \\
\hline 1011 & Procesamiento y conservación de carne y productos cárnicos \\
\hline 1012 & Procesamiento y conservación de pescados, crustáceos y moluscos \\
\hline 1020 & $\begin{array}{l}\text { Procesamiento y conservación de frutas, legumbres, hortalizas y tubércu- } \\
\text { los }\end{array}$ \\
\hline 1030 & Elaboración de aceites y grasas de origen vegetal y animal \\
\hline 1040 & Elaboración de productos lácteos \\
\hline 1062 & Descafeinado, tostión y molienda del café \\
\hline 1063 & Otros derivados del café \\
\hline 1071 & Elaboración y refinación de azúcar \\
\hline 1072 & Elaboración de panela \\
\hline 1082 & Elaboración de cacao, chocolate y productos de confitería \\
\hline \multicolumn{2}{|c|}{ Elaboración de bebidas } \\
\hline 1104 & $\begin{array}{l}\text { Elaboración de bebidas no alcohólicas, producción de aguas minerales y de } \\
\text { otras aguas } \\
\text { Embotelladas }\end{array}$ \\
\hline
\end{tabular}




\begin{tabular}{|c|c|}
\hline \multicolumn{2}{|c|}{ Fabricación de productos textiles } \\
\hline 1311 & Preparación e hilatura de fibras textiles \\
\hline 1391 & Fabricación de tejidos de punto y ganchillo \\
\hline \multicolumn{2}{|c|}{ Confección de prendas de vestir } \\
\hline 1410 & Confección de prendas de vestir, excepto prendas de piel \\
\hline \multicolumn{2}{|c|}{$\begin{array}{l}\text { Transformación de la madera y fabricación de productos de madera y de corcho, excepto } \\
\text { muebles; fabricación de artículos de cestería y espartería }\end{array}$} \\
\hline 1620 & $\begin{array}{l}\text { Fabricación de hojas de madera para enchapado; fabricación de tableros } \\
\text { contrachapados, tableros laminados, tableros de partículas y otros tableros } \\
\text { y paneles }\end{array}$ \\
\hline 1630 & $\begin{array}{l}\text { Fabricación de partes y piezas de madera, de carpintería y ebanistería para } \\
\text { la construcción }\end{array}$ \\
\hline \multicolumn{2}{|c|}{ Fabricación de papel, cartón y productos de papel y cartón } \\
\hline 1701 & Fabricación de pulpas (pastas) celulósicas; papel y cartón \\
\hline 1702 & $\begin{array}{l}\text { Fabricación de papel y cartón ondulado (corrugado); fabricación de enva- } \\
\text { ses, empaques y de embalajes de papel y cartón }\end{array}$ \\
\hline
\end{tabular}

Fuente: Clasificación Industrial Internacional Uniforme (CIIU)

El estudio se aplicó a 15 empresas de Manizales y la muestra para la aplicación del instrumento es de 12 empresas. La precisión de la muestra final se caracteriza por un error máximo de 5 puntos porcentuales con un nivel de confianza del $95 \%$.

\section{Medición del estado innovador}

La medición del estado innovador en empresas medianas y pequeñas agroindustriales se llevó a cabo a través del diseño de un cuestionario con valoración a través de escala de Likert, en la cual la persona entrevistada valoraba 7 factores en una escala de 1 a 5 . En la tabla 3 se discriminan estos ítems. 
Tabla 3. Medición del estado innovador

\begin{tabular}{|c|c|}
\hline Factor & Aspectos a considerar \\
\hline $\begin{array}{c}\text { Estado } \\
\text { Innovador }\end{array}$ & $\begin{array}{l}\text { - Introducción de nuevos productos al mercado } \\
\text { - Mejoras significativas en los productos de la empresa - ajustes en los } \\
\text { atributos y componentes de los productos- } \\
\text { - Actualización y/o renovación en los equipos } \\
\text { - Nuevo proceso o sistema de fabricación o producción - técnicas, materia- } \\
\text { les, suministros, programas- } \\
\text { - Actualizaciones o modificaciones sustanciales en los métodos organiza- } \\
\text { tivos -sistema de gestión del conocimiento, en la organización del lugar de } \\
\text { trabajo, o en la gestión de las relaciones internas de la empresa- } \\
\text { - Nuevas o mejoras significativas en técnicas y métodos de mercadeo } \\
\text { - Nuevas técnicas de comercialización -canales para promoción y ventas, o } \\
\text { modificaciones significativas en el empaque o diseño del producto- }\end{array}$ \\
\hline
\end{tabular}

Fuente: Estructuración basada en clasificación de innovación OCDE (2005).

\section{Medición de la percepción de la efectividad}

En la presente investigación, la medida de percepción de efectividad está basada en un test que recoge 10 de las 13 dimensiones propuestas por Gupta y Govindarajan (1984), se descartan las dimensiones de "asuntos políticos y públicos" "reducción en el abandono voluntario de los trabajadores” y "ausentismo laboral” por el tipo de estructura empresarial y cultura corporativa (ver Tabla 4).

Tabla 4. Medición percepción de efectividad

\begin{tabular}{|c|c|}
\hline Factor & Aspectos a considerar \\
\hline $\begin{array}{c}\text { Percepción de } \\
\text { Efectividad }\end{array}$ & $\begin{array}{l}\text { - Calidad de sus productos } \\
\text { - Coordinación de procesos internos } \\
\text { - Mejoras en las tareas asignadas al personal } \\
\text { - Satisfacción de los clientes frente a sus productos } \\
\text { - Adaptación a las necesidades cambiantes de los mercados } \\
\text { - Imagen de la empresa y de sus productos } \\
\text { - Participación en el mercado } \\
\text { - Rentabilidad } \\
\text { - Nivel de productividad } \\
\text { - Motivación de empleados para el ejercicio de sus labores }\end{array}$ \\
\hline
\end{tabular}

Fuente: Gupta y Govindarajan (1984) 


\section{Resultados}

Los resultados se presentan conforme a los objetivos trazados en tres apartados. En el primero se encuentra el estado innovador de las empresas medianas y pequeñas agroindustriales de Manizales; el segundo, identifica la percepción de desempeño en las empresas objeto de estudio; en el último apartado, se hace referencia a la relación entre el estado innovador hallado y la percepción de desempeño.

\section{Estado inovador de las empresas medianas y pequeñas agroindustriales de Manizales}

De las 12 empresas medianas y pequeñas agroindustriales de Manizales objeto de estudio, se evidencia que el 58, 3\% corresponden al sector de confecciones de prendas de vestir (CIIU 1410), el resto se distribuye en el sector de procesamiento y conservación de cárnicos (8,3\%); procesamiento y conservación de frutas, legumbres entre otros (8,3\%); elaboración de panela (8,3\%); elaboración de productos lácteos $(8,3 \%)$ y fabricación de partes de piezas de madera para la construcción $(8,3 \%)$.

Una vez obtenidos los resultados, se puede determinar que las pymes agroindustriales de Manizales presentan mayor innovación en procesos, específicamente, en la actualización o renovación de equipos, seguido de la innovación en producto -mejoras en los productos o ajustes en éstos- y por último en innovación en organización con cambios en la gestión. (Ver tabla 5).

Tabla 5. Estado Innovador en las pymes agroindustriales

\begin{tabular}{|c|c|c|}
\hline Factor & Items & Media \\
\hline \multirow{5}{*}{$\begin{array}{l}\text { Estado } \\
\text { Innovador }\end{array}$} & Introducción de nuevos productos al mercado & 2,75 \\
\hline & $\begin{array}{l}\text { Mejoras significativas en los productos de la empresa - ajus- } \\
\text { tes en los atributos y componentes de los productos- }\end{array}$ & 3,67 \\
\hline & Actualización y/o renovación en los equipos & 4,33 \\
\hline & $\begin{array}{l}\text { Nuevo proceso o sistema de fabricación o producción - técni- } \\
\text { cas, materiales, suministros, programas- }\end{array}$ & 3,50 \\
\hline & $\begin{array}{l}\text { Actualizaciones o modificaciones sustanciales en los méto- } \\
\text { dos organizativos -sistema de gestión del conocimiento, en } \\
\text { la organización del lugar de trabajo, o en la gestión de las } \\
\text { relaciones internas de la empresa- }\end{array}$ & 3,58 \\
\hline
\end{tabular}




\begin{tabular}{|c|c|c|}
\hline Factor & Items & Media \\
\hline \multirow{2}{*}{$\begin{array}{l}\text { Estado } \\
\text { Innovador }\end{array}$} & $\begin{array}{l}\text { Nuevas o mejoras significativas en técnicas y métodos de } \\
\text { mercadeo }\end{array}$ & 3,50 \\
\hline & $\begin{array}{l}\text { Nuevas técnicas de comercialización -canales para promo- } \\
\text { ción y ventas, o modificaciones significativas en el empaque o } \\
\text { diseño del producto- }\end{array}$ & 3,50 \\
\hline
\end{tabular}

Fuente: Elaboración propia

Asimismo, en la Tabla 5 se aprecia que son pocas las empresas objeto de estudio que innovan con la introducción de nuevos productos $(2,75)$; se puede considerar que la mayoría de las pymes agroindustriales apuntan al sostenimiento y posicionamiento a través de actividades y procesos productivos, especialmente con la adquisición de equipos para el alcance de calidad y productividad en el mercado.

Por lo anterior, se precisa importante conocer además del estado innovador, la percepción de desempeño de los representantes de las empresas, con el fin de considerar su las variables de competitividad en el contexto.

- Percepción de Desempeño en las empresas medianas y pequeñas agroindustriales de Manizales

La percepción de desempeño manifestada por los representantes de las empresas encuestadas para el estudio, se orienta hacia la calidad de los productos $(4,58)$; es decir, la mayoría de las empresas consideran que es el factor clave para lograr efectividad empresarial, lo cual, es consistente con el aspecto de imagen empresarial y de los productos $(4,50)$ y la participación en el mercado $(4,33)$ (ver tabla 6$)$.

Tabla 6. Percepción de Efectividad en las pymes agroindustriales

\begin{tabular}{|c|l|c|}
\hline Factor & \multicolumn{1}{|c|}{ Items } & Media \\
\hline \multirow{4}{*}{$\begin{array}{c}\text { Percepción de } \\
\text { Efectividad }\end{array}$} & Calidad de sus productos & 4,58 \\
\cline { 2 - 3 } & Coordinación de procesos internos & 4,17 \\
\cline { 2 - 3 } & Mejoras en las tareas asignadas al personal & 3,83 \\
\cline { 2 - 3 } & Satisfacción de los clientes frente a sus productos & 4,17 \\
\cline { 2 - 3 } & Adaptación a las necesidades cambiantes de los mercados & 3,75 \\
\hline
\end{tabular}




\begin{tabular}{|c|l|c|}
\hline Factor & \multicolumn{1}{|c|}{ Items } & Media \\
\hline \multirow{4}{*}{$\begin{array}{c}\text { Percepción de } \\
\text { Efectividad }\end{array}$} & Imagen de la empresa y de sus productos & 4,50 \\
\cline { 2 - 3 } & Participación en el mercado & 4,33 \\
\cline { 2 - 3 } & Rentabilidad & 4,00 \\
\cline { 2 - 3 } & Nivel de productividad & 3,83 \\
\cline { 2 - 3 } & Motivación de empleados para el ejercicio de sus labores & 4,00 \\
\hline
\end{tabular}

\section{Fuente: Elaboración propia}

Por su parte, la adaptación a las necesidades cambiantes del mercado es un factor que no ha contribuido en mayor medida a la efectividad organizacional de las pymes estudiadas $(3,75)$ dada a la concentración de mejorar aspectos internos que posteriormente les permita ser competitivos en el entorno.

Teniendo en cuenta los aspectos estado innovador y percepción de desempeño, a continuación se explica la relación entre los mismos y su contribución a la exigencia competitiva, eficiencia, crecimiento empresarial y capacidades organizacionales.

\section{Relación estado innovador con la percepción de desempeño en las empresas medianas y pequeñas agroindustriales de Manizales}

Para determinar la relación entre el estado innovador y percepción de efectividad como medida de desempeño organizacional, se realizó una correlación canónica (Bishop, Feinberg y Holland, 1975) que arrojó un coeficiente de bondad de ajuste 1,778 . Éste, permite concluir que la relación entre los conjuntos de variables es muy alta, produciendo una pérdida de solo 0,222 respecto a la correlación perfecta (2.00). Es decir, se confirma la relación entre estado innovador con la percepción de desempeño en las empresas medianas y pequeñas agroindustriales de la ciudad de Manizales (Ver tabla 7). 
Tabla 7. Relación estado innovador y percepción de efectividad

\begin{tabular}{|c|c|c|c|c|c|c|c|c|c|c|}
\hline \multicolumn{11}{|c|}{ Ajuste } \\
\hline & \multirow{3}{*}{ Establecer } & \multicolumn{3}{|c|}{ Ajuste múltiple } & \multicolumn{3}{|c|}{ Ajuste múltiple } & \multicolumn{3}{|c|}{ Pérdida simple } \\
\hline & & \multicolumn{2}{|c|}{ Dimensión } & \multirow{2}{*}{ Suma } & \multicolumn{2}{|c|}{ Dimensión } & \multirow{2}{*}{ Suma } & \multicolumn{2}{|c|}{ Dimensión } & \multirow{2}{*}{ Suma } \\
\hline & & 1 & 2 & & 1 & 2 & & 1 & 2 & \\
\hline \multirow{7}{*}{ 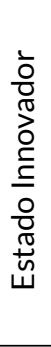 } & Prodnuevosa &, 547 & ,142 & ,690 & ,538 & , 137 & ,676 & ,009 &, 005 &, 014 \\
\hline & Mejorasproda & 1,188 & , 100 & 1,288 & 1,180 & 095 & 1,275 & ,008 &, 005 & ,012 \\
\hline & Equiposa ${ }^{a}$ & 863 & ,649 & 1,512 &, 862 & ,648 & 1,511 & ,001 & ,001 & ,002 \\
\hline & Procesosa $^{a}$ & , 147 &, 317 &, 464 & ,145 & ,314 &, 459 &, 002 & ,003 & ,005 \\
\hline & Organizaciona $^{a}$ &, 434 & ,886 & 1,320 &, 432 & 884 & 1,316 & 002 &, 002 &, 003 \\
\hline & Mercadeoa $^{a}$ &, 900 &, 267 & 1,167 & 893 &, 265 & 1,158 & ,007 & ,002 &, 009 \\
\hline & Comercializaciona $^{a}$ &, 377 & ,124 &, 501 &, 374 & ,123 & ,496 & 004 & ,001 &, 005 \\
\hline \multirow{10}{*}{ 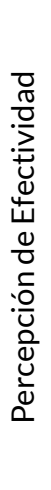 } & Calidadproda &, 497 &, 046 &, 543 &, 497 &, 046 &, 543 & , 000 & 0,000 &, 000 \\
\hline & Mejorprocesosaa & ,014 & ,566 & ,580 & ,013, & ,557 & ,569 & 001 & 010, & ,011 \\
\hline & Mejortareasa ${ }^{a}$ & ,270 & ,390 & ,660 & ,263 & ,364 & 627, & 008 & ,026 & ,033 \\
\hline & Satisfclientesa ${ }^{a}$ &, 160 & ,637 &, 798 & , 160 & ,637 & ,798 &, 000 &, 000 &, 000 \\
\hline & Adaptamercadosa ${ }^{a}$ &, 800 &, 119 &, 919 & 800 &, 118 &, 918 &, 000 &, 001 &, 001 \\
\hline & Imagenempa &, 255 & ,032 & ,287 & ,255 & ,032 & ,287 & ,000 &, 000 &, 000 \\
\hline & Participmercadoa & 1,049 & 081 & 1,130 & 1,048 &, 075 & 1,123 &, 001 &, 006 &, 006 \\
\hline & Rentabilidada ${ }^{a}$ &, 026 & 1,146 & 1,171 & 013 & 1,146 & 1,158 & ,013 &, 000 &, 013 \\
\hline & Productividadaa & ,621 &, 416 & 1,037 & ,616 &, 413 & 1,029 & 005 & 003 &, 008 \\
\hline & Motivaemplaa & 1,053 & , 107 & 1,161 & 1,051 &, 077 & 1,129 & 002 & ,030 & ,032 \\
\hline \multicolumn{11}{|c|}{ a. Nivel de escalamiento óptimo: Ordinal } \\
\hline
\end{tabular}

Fuente: elaboración propia

En referencia a los antecedentes expuestos y basados en los análisis de los resultados, se afirman estudios como el de Ortega (2000) quien manifiesta que la innovación tecnológica permite a las pymes dar respuesta a las exigencias competitivas del contexto y lograr índices de eficiencia y competitividad empresarial (Goldenberg, Horowitz, Levav y Mazursky, 2003; Zhan y Li, 2012; Teece, 1996; Cano, Sánchez, González y Pérez, 2014; Davenport, 1993; Freire, 2000; Coronado, Echeverri y Arias, 2014; Lombardi y Mora, 2014).

De esta manera, la acción innovadora en productos, procesos y sistemas de gestión que promueven las pequeñas y medianas empresas tienen un impacto positivo sobre su crecimiento. Es decir, la innovación mejora las capacidades, la 
competitividad, el desempeño y el rendimiento empresarial de las pymes (García, Gálvez y Maldonado, 2016; Lecerf, 2012 y Wang, 2014).

\section{Conclusiones}

El estudio concluye que la economía agrícola de Colombia es considerada factor de oportunidad y desarrollo empresarial. Su contribución a través de materias primas a otros sectores y su aporte al PIB y al empleo nacional es de gran alcance en la mayoría de departamentos, reflejado en los renglones de cultivo de café, cultivo de otros productos agrícolas, producción pecuaria y silvicultura (DANE, 2016). Específicamente, para el departamento de Caldas existe un gran reto con los distritos agroindustriales como estrategia de articulación y aprovechamiento de ventajas propias para propiciar el desarrollo económico de la región basado en los renglones de cultivo de café, plátano, cacao, ganadería, turismo e industria.

El estudio confirma la relación del estado innovador de las pymes agroindustriales de Manizales con el desempeño organizacional. Es decir, las empresas que realizan acciones innovadoras, perciben un mayor desempeño en la gestión de sus organizaciones, reflejado en calidad, imagen de productos y empresa, satisfacción del consumidor, participación en el mercado y coordinación de procesos internos. Lo anterior ratifica a la innovación como estrategia para la adaptación a los contextos, sostenimiento del desempeño y éxito empresarial (Damanpour y Gopalakrishnan, 2001; Afcha, 2011; Camisón y Villar, 2014).

La mayoría de las empresas agroindustriales de la ciudad de Manizales introduce el factor innovador para su crecimiento y posicionamiento en el mercado, de allí que procuren por la innovación en procesos que les garanticen eficiencia y su continuidad en el entorno competitivo, confirmando los sustentado en los estudios de Dankbaar y Cobbenhagen (2000); Montoya (2004); Parra, Mesa, Corrales y Aguirre (2007); Davenport (1993); Freire (2000); Manual de Oslo, OCDE (2005); Coronado, Echeverri y Arias (2014); Lombardi y Mora (2014), los cuales coinciden que este tipo de innovación desarrolla estrategias para la diversificación y novedad de procesos de manera significativa, aportando mayor valor y logrando mejores niveles competitivos.

En el ámbito colombiano, se pueden contrastar los hallazgos de este estudio con el realizado por Zartha et al. (2009) quienes encontraron que el 50\% de las empresas del sector agroindustrial del departamento de Antioquia, son 
consideradas potencialmente innovadoras en producto-servicio y poseen carencias y menor desarrollo en la innovación en procesos. Caso contrario a lo hallado en Manizales en este mismo sector para las pymes, en el que los resultados indican que éstas presentan mayor innovación en procesos de actualización o renovación de equipos principalmente -en menor medida de nuevos procesos o sistemas de fabricación o producción- técnicas, materiales, suministros, programas, seguido de la innovación en producto.

La innovación en producto -en relación con la introducción de nuevos productos al mercado- es baja en el tipo de industrias estudiadas, podría significar oportunidades para el desarrollo de estrategias en este sector que logren la diversificación y novedad de productos o sus atributos de manera significativa, aportando mayor valor y logrando mejores niveles competitivos. Lo anterior, soportado por políticas que mejoren las condiciones productivas e impulsen los desarrollos innovadores; para ello, es necesario contar con recursos, conocimientos y capacidades que estén acordes con los objetivos de las organizaciones (Teece, 1996; Cano, Sánchez, González y Pérez, 2014).

Si bien se determina que existe una relación significativa entre innovación y desempeño -lo que implica que las acciones innovadoras tienen alto impacto para la productividad organizacional- (Knigth, 1967; Dosi, 1988; Sánchez, García y Mendoza, 2014), las pymes del sector deben mejorar aspectos como los métodos de mercadeo, dado que éstos optimizan el cumplimiento de expectativas del mercado y propenden por el logro de una alta rentabilidad y productividad, tal como lo demostrado por García, Quintero y Arias (2014) en su estudio, en el que concluyen que las capacidades de innovación relacionadas con el cliente y mercadeo influyen en el desempeño organizacional.

Finalmente, debido a la alta importancia social y económica del sector agroindustrial para el país y para el departamento, este tipo de estudios aportan insumos diagnósticos para los programas nacionales que fomentan el desarrollo agroindustrial como El Sistema Nacional de Ciencia y Tecnología Agroindustrial y El Sistema Nacional de Competitividad, Ciencia, Tecnología e Innovación, con el fin de que continúen desarrollando modelos para la solución de problemáticas del sector agropecuario, que permitan mejorar los niveles de productividad y competitividad para las empresas, contribuyendo al mejoramiento de su desempeño y gestión empresarial. 


\section{Referencias}

Afcha, S. (2011). Innovaciones organizacionales y su efecto sobre el desempeño empresarial. Revista Venezolana De Gerencia, (56), 544-563.

Afuah, A. (1998). Innovation Management: Strategies, Implementation, and Profits. Oxford: Oxford University Press.

Argoti, A.C. (2009). Las pymes agroindustriales de Nariño y su aporte en el desarrollo de la región. Revista de la Facultad de Ciencias Económicas y Administrativas Universidad de Nariño, 10(1), 7-34.

Austin, J. E. (1992). Agroindustrial project analysis: Critical design factors. Baltimore: Johns Hopkins University Press.

Barbosa, B. y Dominique, S. (2012). La innovación de los procesos: diferenciación en los servicios turísticos. Estudios y Perspectivas en Turismo, 21(4), 963-976.

Bishop, Y., Feinberg, S. y Holland, P. (1975). Discrete multivariate analysis Cambridge, MA: MIT Press.

Camisón, C. y Villar, A. (2014). Organizational innovation as an enabler of technological innovation capabilities and firm performance. Journal of Business Research, (67), 2891-2902.

Campbell, J. (1990). Modeling the performance prediction problem in industrial and organizational psychology. Handbook of industrial and organizational psychology, 1, 687-732.

Cano, M., Sánchez, G., González, M. y Pérez, J. (2014). El rol del capital intelectual en la innovación de las empresas. European Scientific Journal, 10(28), 348-366.

Cárdenas, M. (1999). Colombia: un sindicalismo errante tantea su futuro. En L. Arango, G. y C. M López. Globalización, Apertura Económica y Relaciones Industriales en América Latina (Comp.). Bogotá, Colombia: Centro de Estudios Sociales, CES, Universidad Nacional de Colombia. 
Cardona A, Castiblanco M., y Díaz, S. (2013). Innovación empresarial: una mirada desde la competitividad, el desarrollo local y la transformación productiva para la internacionalización en Colombia. Semestre Económico, 16(34), 149-168.

Coronado, A., Echeverri, A. y Arias, J. (2014). Aproximación a la cooperación en innovación en empresas del Programa de Asociatividad y Desarrollo Empresarial Sectorial -PADES- en Antioquia (Colombia). Revista de la Facultad de Ciencias Económicas de la Universidad Militar Nueva Granada, 22(2), 185-205.

Cortés, E. (2007). La agroindustria y viabilidad del sector agropecuario. Revista CES Medicina Veterinaria y Zootecnia, 2(1), 74-80.

Cortes, E., Suárez, H. y Pardo, S. (2008). Producción sostenible en la agricultura Colombiana. Ingeniería de Recursos Naturales y del Ambiente, 7, 48-56.

Daft, R. (2007). Estrategia, diseño organizacional y efectividad. En Teoría y $D i$ seño Organizacional (pp.70-79). México, D.F., México: Cengage Learning Editores.

Damanpour, F. (1991). Organizational Innovation: A Meta-analysis of Effects of Determinants and Moderators. Academy of Management Journal, 34(3), 555-590.

Damanpour, F. y Evan, W. (1984). Organizational innovation and performance: the problem of organizational lag. Administrative Science Quarterly, 29(3), 392-409.

Damanpour, F. y Gopalakrishnan, S. (2001). The dynamics of the adoption of products and process innovations in organizations. Journal of Management Studies, 38(1), 45-65.

Dankbaar, B. y Cobbenhagen, J. (2000). Learning and collaboration in practice. En F. Boekema, K. Morgan, S. Bakkers y R. Rutten (Eds.). Knowledge, innovation and economic growth: The theory and practice of learning regions ( $\mathrm{p}$. 266) Cheltenham (UK): Edward Elgar.

Davenport, T. (1993). Process innovation, reengineering work through information technology. Boston, Estados Unidos: Harvard Business School Press. 
Departamento Nacional de Estadísticas, DANE (2016). Cuentas nacionales anuales. Recuperado de: http://www.dane.gov.co/index.php/cuentas-economicas/ cuentas-trimestrales.

Departamento Nacional de Planeación, DPN. (2007). Agenda interna para la productividad y la competitividad-Documento regional, Caldas-. Bogotá.

Dosi, G. (1988). Sources, procedures and microeconomic effects of innovation. Journal of Economic Literature, 26, 1120-1171.

Evan, W. (1966). Organizational Lag. Human Organization, (25), 51-53.

Freire, A. (2000). Inovação: novos produtos, serviços e negócios para Portugal. Lisboa, Portugal: Editorial Verbo.

García, O., Quintero, J. y Arias, J. (2014). Capacidades de innovación, desempeño innovador y desempeño organizacional en empresas del sector servicios. Cuadernos de Administración, 27(49), 87-108.

García, D., Gálvez, E. y Maldonado, G. (2016). Efecto de la innovación en el crecimiento y el desempeño de las Mipymes de la Alianza del Pacífico, Un estudio empírico. Estudios Gerenciales. 1-10.

Gobernación de Caldas (2001). Plan de Desarrollo Departamental 2001-2003. Ordenanza número 243. Recuperado de http://www.gobernaciondecaldas.gov. co/web/media/pdf/planeacion/ORDENANZA_723_-_POR_MEDIO_ DE_CUAL_SE_ADOPTA_EL_PLAN_DESARROLLO_2013-2015.pdf

Goldenberg, J., Horowitz, R., Levav, A. y Mazursky, D. (2003). Finding your innovation sweet spot. Harvard Business Review, 81(3), 3-10

González, J. (2013). Innovar: un imperativo para competir en la economía mundial. Revista de Ingeniería, 38, 52-59.

Gupta A. y Govindarajan V (1984). Business Unit Strategy, Managerial Characteristics, and Business Unit Effectiveness at Strategy Implementation. Academy of Management Journal, 27(1), 25-41. 
Ickis, J., Leguizamón, F., Metzger, M. y Flores, J. (2009). La agroindustria: campo fértil para los negocios inclusivos. Revista Latinoamericana de Administración, (43) 107-124

Knight, K. (1967). A descriptive model of the intra-firm innovation process. Journal of Business, 4O(4), 478-496.

Köhler, H. y González, S. (2014). Elementos para un concepto sociológico de innovación. Revista de Metodología de las Ciencias Sociales, (29) 67-88.

Lecerf, M. (2012). Internationalization and innovation: The effects of a strategy mix on the economic performance of French SMEs. International Business Research, 5(6), 2-13.

Likert, R. (1932). A technique for development of attitude scales. Archives of Psychology, (140), 44-53.

Lombardi, G. y Mora, J. (2014). Determinantes de la innovación en productos o procesos: el caso colombiano. Revista De Economía Institucional, 16 (31), 251-262.

Medina, J., Vélez, A., Zartha, J., Ruiz, S. y Copete, H. (2012). ADN de la innovación - de factores claves de innovación en 21 empresas del sector agroindustrial. Biotecnología en el Sector Agropecuario y Agroindustrial, 1O(2), 197206.

Molnar J. y Rogers D. (1976). Organizational Effectiveness: An Empirical Comparision of the Goal and System Resource Approaches. The Sociological Quarterly, 17(3), 401-413.

Montoya, O. (2004). Schumpeter, innovación y determinismo tecnológico. Scientia et Technica, 10(25), 209-213.

Organización para la cooperación y el desarrollo económico, OCDE (2005). Manual de Oslo: Guía para la recogida e interpretación de datos sobre la innovación. Recuperado de: http://www.uis.unesco.org/Library/Documents/ OECDOsloManualo5_spa.pdf 
Organización de las Naciones Unidas para la Alimentación y la Agricultura, FAO (1997). El Estado Mundial de la Agricultura y la Alimentación. Recuperado de http://www.fao.org/docrep/w5800s/W5800s00.htm

Ortega, J. (2000). Gestión de La tecnología, innovación y competitividad empresarial. Ingeniería y competitividad, 2(1), 7-11.

Ovalle, A., Martínez, E., Ramírez, l. y Restrepo, J. (2012). Relación de la norma técnica colombiana Icontec NTC5801 con los procesos de gestión de la innovación en las pymes del sector textil y de confección de Manizales-Colombia. Revista ingeniería industria, 11(2), 57-70.

Parra, R., Mesa, J., Corrales, M. y Aguirre, M. (2007). La auditoría de la innovación. Medellín, Colombia: Fondo Editorial Universidad EAFIT.

Planella, I. (1981). La agroindustria en Colombia (Una revisión y análisis bibliográfico). Bogotá, Colombia: Instituto Interamericano de Cooperación, IICA-OEA.

Proexport (2012). Sector Agroindustrial Colombiano. Bogotá, Colombia.

Quinn R. y Rohrbaugh J. (1983). Spatial model of effectiveness criteria: Towards a competing values approach to Organizational analysis. Management science, 29(3), 363-377.

Rose, S., Shipp, S., Lal, B. y Stone, A. (2009). Frameworks for Measuring Innovation: Initial Approaches. In S. a. T. P. Institute (Ed.) (Vol. 06). Washington DC: Athena Alliance.

Rotundo, M. y Sackett, R. (2002). The relative importance of task, citizenship, and counterproductive performance to global ratings of job performance: a policy-capturing approach. Journal Of Applied Psychology, 87(1), 66-80.

Sánchez, D. (2005). Capitalism, development and the state. A critical account of Schumpeter's theory of the state. Revista de Economía Institucional, 7(13), 81-100.

Sánchez, Y., García, F. y Mendoza, E. (2014). Determinantes de la capacidad de innovación regional en México. Una tipología de las regiones. Región y Sociedad, 26(61), 119-160. 
Schumpeter, J. (1934). The theory or economic development. Cambridge, Massachusetts: Harvard University Press.

Teece, D. (1986). Profiting from technological innovation: Implications for integration, collaboration, licensing and public policy. Research Policy, 15(6), 285-305.

Tushman, L. y Anderson, P. (1986). Technological Discontinuities and Organizational Environments. Administrative Science Quarterly, 31(3), 439465 .

Varela, C., Contesse, D. y Silva, P. (2009). Global Entrepreneurship Research. Santiago de Chile: Universidad del Desarrollo.

Wang, C. (2014). A longitudinal study of innovation competence and quality management on firm performance. Innovation: Management, Policy y Practice, $16(3), 392-403$.

Yamakawa, P. y Ostos, J. (2011). Relación entre innovación organizacional y desempeño organizacional. Revista Universidad y Empresa, 13(21), 93-115.

Yoguel, G., Barletta, F. y Pereira, M. (2013) De Schumpeter a los postschumpeterianos: viejas y nuevas dimensiones analíticas. Problemas del Desarrollo, 174(44), 35-59.

Zartha, J.,Ávalos, A., Aguilar, S. y Castrillón, F.(2009). Metodología para la medición de innovaciones tecnológicas aplicadas a empresas del sector agroindustrial. Biotecnología en el Sector Agropecuario y Agroindustrial, 7(2), 89-98.

Zhang, Y. y Li, H. (2010) Innovation search of new ventures in a technology cluster: The role of ties with service intermediaries. Strategic Management Journal, 31(1), 88-109. 